\title{
Comparative effectiveness study of chemotherapy in follicular lymphoma patients in the rituximab era: a Japanese claims database study
}

\author{
Saaya Tsutsué*,1 (iD), Kensei Tobinai², Jingbo $\mathrm{Yi}^{3}$ \& Bruce Crawford ${ }^{3}$ \\ ${ }^{1}$ Celgene K.K., a Bristol Myers Squibb Company, JP Tower, 2-7-2 Marunouchi Chiyoda-ku, Tokyo 100-7010, Japan \\ ${ }^{2}$ National Cancer Center Hospital, Tokyo, Japan \\ ${ }^{3}$ Syneos Health, Tokyo, Japan \\ *Author for correspondence: Tel.: +81 352240 547; saaya.tsutsue@bms.com
}

\begin{abstract}
Aim: To evaluate comparative effectiveness of rituximub (R)-based versus non-R-based therapies for follicular lymphoma patients in Japan, where limited studies have been reported. Materials $\&$ methods: Patients who received R-based index regimens were propensity score matched to those who did not receive $R$, based on patient baseline attributes and clinical characteristics using Japanese retrospective claims database to assess clinical and economic outcomes. Results \& conclusion: A total of 1947 patients remained in the overall follicular lymphoma cohorts: 1294 receiving an R-based and 653 a non-R-based regimen. Patients on R-based therapy underwent fewer hospitalizations and had a shorter length of stay, but had higher costs during the first year of intensive R-based therapy. Improved clinical outcomes were associated with patients who were younger, female and chose R-based regimens in first index line.
\end{abstract}

First draft submitted: 14 August 2020; Accepted for publication: 22 September 2020; Published online: 6 October 2020

Keywords: comparative effectiveness - health economics - hematologic/lymphoma • pharmacoeconomics - real-world evidence

Follicular lymphoma (FL), which belongs to the subtype of non-Hodgkin lymphomas (NHL), accounts for approximately $20 \%$ of all lymphomas in the USA [1]. In Japan, FL is the second most common subtype of NHL, accounting for $13.5 \%$ of all NHL types, followed by diffuse large B-cell lymphoma [2,3]. In addition, FL is associated with approximately $30 \%$ lifetime risk of transforming to a more aggressive lymphoma, most commonly to diffuse large B-cell lymphoma $[4,5]$.

The availability of rituximab (R) for FL patients in Japan since 2001 has substantially changed FL therapy, providing multiple treatment options for FL patients beyond 'watch and wait' and stem cell transplantation (SCT) [6]. Recommended treatment options for FL include R-based therapies (e.g., R plus bendamustine; R plus cyclophosphamide, doxorubicin, vincristine and prednisone; R plus cyclophosphamide, vincristine and prednisone or R-monotherapy). A recent clinical review [7] underscores the need to tailor treatment based on an individual patient's disease state, risk status and genetic background for FL. There is no consensus for treatment of FL patients associated with multiplicity of relapse in course of disease progression. Therefore, the real-world evidence to clarify current treatment patterns, treatment-based economic and clinical outcomes on standard treatments is needed from comparative effectiveness perspective.

Five-year overall survival (OS) reported for FL patients in UK as 75.6\% of Haematological Malignancy Research Network data, covering the period from 2007 to 2015 ( $\mathrm{n}=923$ patients) [8]. In a database study of patients with FL from the USA ( $\mathrm{n}=1346$ ) between 2008 and 2015, 2-year OS was reported as 86.9\% after first-line (1L) treatment [9]. The economic burden of FL in the USA has been reported to be relatively high, with mean monthly costs per patient of US $\$ 10,460$ (USD) in total, including $\$ 6482$ of FL-related costs, based on a database study of 1595 patients who received 1L treatment for FL between 2008 and 2015 [10]. That study reported that over half of the patients (51.0\%) had $\geq 1$ inpatient visit and $57.2 \%$ had $\geq 1$ emergency department visit.

Future Medicine 


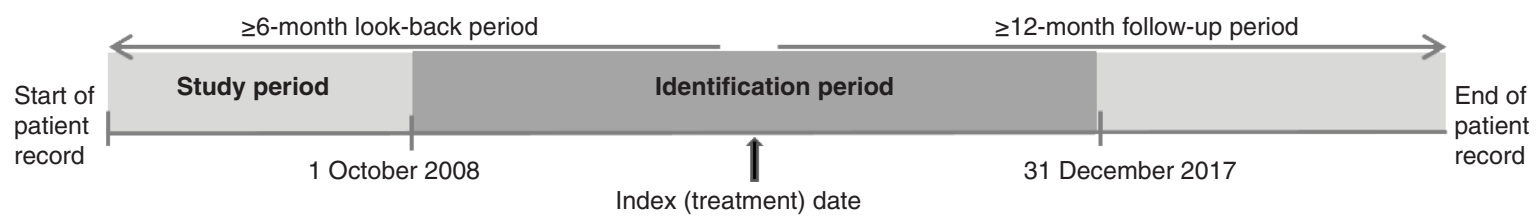

Figure 1. Description of study and identification periods.

To date, however, there have been no comprehensive published studies describing treatment patterns, economic burden and survival outcomes of FL patients in Japan. Therefore, the objectives of our study are to describe treatment patterns, healthcare resource utilization (HCRU) and costs during each year of therapy for FL in Japan. Additionally, the study also aims to evaluate OS and time-to-next-treatment (TTNT) outcomes from the start of $1 \mathrm{~L}$ treatment to better understand the clinical landscape for FL patients.

\section{Materials \& methods}

\section{Database \& patient selection}

Data for this administrative claims data study were analyzed using the Medical Data Vision (MDV) database (http://www.mdv.co.jp/), a Diagnosis Procedure Combination database comprising anonymized inpatient MDV database records and outpatient data from over 400 Japanese hospitals, covering approximately $22 \%$ of acute phase hospitals (including 187 cancer therapeutic facilities) and data for 26.77 million patient records as of December 2017. The identification period was from 1 October 2008 through 31 December 2017, with a minimum look-back period of 6 months by following previous MDV database study [3] and a minimum follow-up period 12 months relative to the patient index date, which from the start of $1 \mathrm{~L}$ (defined as the first date of systemic treatment during the identification period). The identification and study periods are shown in Figure 1. Our study period continued until 31 December 2018 as last timing for patients who were still on the claims database record during the entire follow-up period based on our criteria of the 12 months minimum follow-up period.

The initial patient cohort included in the analysis were those treated for FL during the identification period and who had a claim with International Classification of Diseases (ICD)-10 diagnosis code for FL (C82.x) on the index date or during the 6-month look-back period. Patients excluded were those who did not have at least two claims (for any disorder) with at least 1 -year follow-up (one claim every 6 months, unless they died within the first 6 months) or who did not have at least one claim (for any disorder) with a 6-month look-back period.

This initial cohort was further narrowed down through propensity score matching (PSM) using covariates as presented by Albarmawi et al. [11]. The covariates included patient demographic characteristics are based on age group ( $<66,66-70,71-75,76-80,81-85,86+$ years), sex, valid measure of disease severity including Charlson comorbidity index score (CCI) and index year (binary variable; before or after 27 October 2010, which was the launch date of bendamustine in Japan). Age and CCI group cutoffs were based similar cutoffs as presented by Albarmawi et al. [11] and slightly modified to include the elderly population in Japan and to account for the patients who by definition had a baseline CCI score of 2 (their FL diagnosis prior to treatment initiation contributes a score of 2).

PSM algorithm [12] was used to eliminate the potential selection bias from baseline covariates for making comparison between $\mathrm{R}$ and non- $\mathrm{R}$-based index regimens. PSM was completed with a 2:1 ratio ( $\mathrm{R}$ vs non- $\mathrm{R}$-based for index regimen) using the nearest-neighbor algorithm and calipers of width equal to 0.2 of the standard deviation of the logit of the propensity score with evaluation for each covariate using standardized mean difference of 0.1 . A 2:1 ratio was chosen based on the number of patients in each group before the PSM to ensure that the non-R-based patients can also be sufficiently included in the match.

\section{Ethics approval \& consent to participate}

This was a retrospective study carried out using an existing hospital claims database with no primary data collection. Patients were informed that their data would be used for research (opt-out system) and their data were de-identified before addition to the database. Data from this database occur in an unlinked fashion. As the data had been anonymized, the Ethical Guidelines for Epidemiological Research in Japan, which require ethics approval and informed consent, are not applicable to this study. Based on the Ethical Guidelines on Biomedical Research Involving Human Subjects, pharmaco-epidemiological studies conducted on medical databases constitute research 
carried out on pre-existing material and information not requiring any interaction with patients [13] and therefore obtaining written informed consent from patients was not required.

\section{Outcome measures \& data analyses}

The outcome measures included: the proportion of patients who received either R-based or non-R-based FL therapy regimens within 30 days of the patient index date; treatment pattern; HCRU, including the use of radiation therapy and SCT; direct medical costs; and survival outcomes including OS and TTNT. R-based regimen categories during $1 \mathrm{~L}$ included $\mathrm{R}$ plus cyclophosphamide, doxorubicin, vincristine and prednisone, $\mathrm{R}$ plus cyclophosphamide, vincristine and prednisone, R-bendamustine, other R-based chemotherapy and R-monotherapy. non-R-based regimens included any chemotherapy without $\mathrm{R}$, immunotherapy/endocrine therapy and other treatments without $\mathrm{R}$ that were not otherwise specified. The first line captured during the identification period was designated as $1 \mathrm{~L}$; this regimen consisted of all drugs received during 30 days before and after the index date. Patients were considered to have a new line of therapy if they received a drug not included in the initial treatment regimen or had a gap of more than 90 days between any treatments. Measures of HCRU included number of outpatient visits, number of hospitalizations, length of hospital stay and number of emergency room visits during each year of follow-up.

In our study, nominal direct medical costs were obtained in Japanese yen (JPY) directly from the database and were totaled during the study period and then stratified by inpatient/outpatient status, cancer drug costs and other pharmacy costs. Direct unadjusted (nominal) medical costs are presented after converting from JPY to USD using the exchange rate based on the first month of every year in Supplementary Table 3. Direct unadjusted (nominal) medical costs are then adjusted to direct adjusted medical costs with regard to Japanese inflation rate based on the calendar year average of Consumer Price Index by the reference year of 2018 [14,15].

OS was also calculated from the patient index date for all patients and stratified by demographic factors (i.e., age, sex and CCI) and treatment groups. In addition, TTNT was calculated from the index date to the next line of therapy, accounting for death as a competing risk.

Continuous variables that follow an approximately normal distribution were summarized using mean, standard deviation $(\mathrm{SD})$, median, minimum and maximum. Categorical variables were summarized using frequency and percentage. For HCRU outcomes, differences between treatment groups were assessed using $t$-tests. For cost outcomes, differences between treatment groups were assessed using gamma generalized linear models with loglink function. For OS, Kaplan-Meier (KM) survival curves and their corresponding median and 95\% CIs were generated for all patients and stratified by the specified groups. Corresponding median and $95 \% \mathrm{CI}$ for hazard ratios were similarly generated using a cumulative incidence model with death as a competing risk from the Fine-Gray model $[16,17]$. Cumulative incidence function curves were also generated. All data analyses were performed using SAS ${ }^{\circledR}$ software version 9.4 or higher.

\section{Results}

\section{Patient demographics \& comorbidities}

During the study identification period of 1 October 2008 through 31 December 2017, a total of 5037 patients were treated for NHL. Of these patients, 3928 had an FL diagnosis on the index date or during the 6-month look-back period before the index date. The remaining inclusion criteria were applied, which yielded 3593 patients who were subject to PSM. Of this prematched patient group, 2938 received a R-based regimen and 655 received a non-R-based regimen. After matching, 1947 patients remained in the overall patient sample, with 1294 receiving an $\mathrm{R}$-based regimen and 653 receiving a non-R-based regimen. The flow of patient attrition is illustrated in Figure 2. The groups were deemed balanced on all matching covariates as all had an absolute standardized mean difference of $<0.1$.

Patient characteristics are shown in Table 1. The mean (SD) ages for patients with FL treated with R-based and non-R-based therapy were 66.3 (11.0) and 67.1 (10.7) years, respectively. In the R-based group, a total of 720 patients (55.6\%) were $\geq 66$ years of age and $107(8.3 \%)$ were $>80$ years of age. The non-R-based group had 368 patients $(56.4 \%) \geq 66$ years of age and $62(9.5 \%)$ were $>80$ years of age. Almost exactly half of both regimen groups were male. The most common index year for both groups was 2015, with nearly half of patients in both groups initiating treatment on or after 2015. The mean follow-up time (SD) for R-based and non-R-based regimen patients was 1296.2 (685.0) days and 1134.2 (722.5), respectively. More non-R-based regimen patients had a death captured in the database compared with the R-based group (22.1 vs 9.7\%). The most common comorbidities for R-based regimen patients and non-R-based regimen patients (other than malignancy in $\sim 99 \%$ of both groups) 
Table 1. Patient demographics and baseline characteristics (pre- and post propensity score matching).

\begin{tabular}{|c|c|c|c|c|c|}
\hline \multirow[t]{2}{*}{ Characteristic } & \multicolumn{2}{|c|}{ Pre PSM } & \multicolumn{3}{|c|}{ Post PSM } \\
\hline & $\begin{array}{l}R \text { based } \\
(n=2938)\end{array}$ & $\begin{array}{l}\text { Non R based } \\
(n=655)\end{array}$ & $\begin{array}{l}R \text { based } \\
(n=1294)\end{array}$ & $\begin{array}{l}\text { Non R based } \\
(n=653)\end{array}$ & $\begin{array}{l}\text { Standardized mean } \\
\text { difference (post PSM) }{ }^{\dagger}\end{array}$ \\
\hline Mean age, years (SD) & $64.5(10.6)$ & $67.2(10.7)$ & $66.3(11.0)$ & $67.1(10.7)$ & 0.0789 \\
\hline - Minimum, maximum, years & $14.0,94.0$ & $28.0,96.0$ & $23.0,94.0$ & $28.0,96.0$ & \\
\hline \multicolumn{6}{|l|}{ Age group, $\mathrm{n}(\%)$} \\
\hline$-<66$ & $1539(52.4 \%)$ & $285(43.5 \%)$ & $574(44.4)$ & $285(43.6)$ & - \\
\hline$-66-70$ & $546(18.6 \%)$ & $120(18.3 \%)$ & $240(18.5)$ & $120(18.4)$ & \\
\hline$-71-75$ & $401(13.6 \%)$ & $96(14.7 \%)$ & $194(15)$ & $96(14.7)$ & \\
\hline$-76-80$ & $266(9.1 \%)$ & $91(13.9 \%)$ & $179(13.8)$ & $90(13.8)$ & \\
\hline$-81-85$ & $144(4.9 \%)$ & $38(5.8 \%)$ & $69(5.3)$ & $38(5.8)$ & \\
\hline - Over 85 & $42(1.4 \%)$ & $25(3.8 \%)$ & $38(2.9)$ & $24(3.7)$ & \\
\hline \multicolumn{6}{|l|}{ Sex, n (\%) } \\
\hline - Female & $1429(48.6 \%)$ & $325(49.6 \%)$ & $648(50.1)$ & $325(49.8)$ & 0.0061 \\
\hline - Male & $1509(51.4 \%)$ & $330(50.4 \%)$ & $646(49.9)$ & $328(50.2)$ & \\
\hline \multicolumn{6}{|l|}{ Year of index date, $\mathrm{n}(\%)$} \\
\hline $\begin{array}{l}\text { - Before bendamustine date ( } 27 \\
\text { October 2010) }\end{array}$ & $169(5.8 \%)$ & $39(6 \%)$ & $72(5.6)$ & $38(5.8)$ & 0.011 \\
\hline $\begin{array}{l}\text { - After bendamustine date ( } 27 \\
\text { October 2010) }\end{array}$ & $2769(94.2 \%)$ & $616(94 \%)$ & $1222(94.4)$ & $615(94.2)$ & \\
\hline \multicolumn{6}{|l|}{ Baseline CCI, n (\%) } \\
\hline$-0-2$ & $1579(53.74 \%)$ & $292(44.58 \%)$ & $576(44.5 \%)$ & $292(44.7 \%)$ & 0.0065 \\
\hline-3 & $318(10.8 \%)$ & $87(13.3 \%)$ & $170(13.1 \%)$ & $86(13.2 \%)$ & \\
\hline-4 & $491(16.7 \%)$ & $111(16.9 \%)$ & $223(17.2 \%)$ & $111(17.0 \%)$ & \\
\hline$-5+$ & $550(18.7 \%)$ & $165(25.2 \%)$ & $325(25.1 \%)$ & $164(25.1 \%)$ & \\
\hline \multicolumn{6}{|l|}{$\begin{array}{l}\text { Follow-up time (from index date until } \\
\text { death or last patient record), days }\end{array}$} \\
\hline - Mean (SD) & $1316.9(693.7)$ & $1137.9(728.1)$ & $1296.2(685.0)$ & $1134.2(722.5)$ & 0.2301 \\
\hline - Median (Q1, Q3) & $1254.0(827.0,1725.0)$ & $1050.0(548.0,1640.0)$ & $1236.0(802.0,1698.0)$ & $1050.0(548.0,1639.0)$ & \\
\hline \multicolumn{6}{|l|}{$\begin{array}{l}\text { Other prior or concurrent primary } \\
\text { cancer }\left(\text { C00-C96, except }\left(77-C 89^{\ddagger}\right), n\right. \\
(\%)\end{array}$} \\
\hline - Yes & $391(13.3 \%)$ & $163(24.9 \%)$ & $192(14.8)$ & $162(24.8)$ & 0.2521 \\
\hline - No & $2547(86.7 \%)$ & $492(75.1 \%)$ & $1102(85.2)$ & $491(75.2)$ & \\
\hline \multicolumn{6}{|l|}{ Comorbidities, n (\%) } \\
\hline - Congestive heart failure & $373(12.7 \%)$ & $116(17.7 \%)$ & $211(16.3)$ & $116(17.8)$ & 0.0388 \\
\hline - Dementia & $16(0.5 \%)$ & $6(0.9 \%)$ & $9(0.7)$ & $6(0.9)$ & 0.025 \\
\hline - Chronic pulmonary disease & $426(14.5 \%)$ & $139(21.2 \%)$ & $241(18.6)$ & $137(21.0)$ & 0.0591 \\
\hline - Rheumatologic disease & $58(2 \%)$ & $15(2.3 \%)$ & $31(2.4)$ & $15(2.3)$ & 0.0065 \\
\hline - Mild liver disease & $550(18.7 \%)$ & $134(20.5 \%)$ & $278(21.5)$ & $133(20.4)$ & 0.0274 \\
\hline - Diabetes with chronic complications & $59(2 \%)$ & $19(2.9 \%)$ & $34(2.6)$ & $19(2.9)$ & 0.0172 \\
\hline - Hemiplegia or paraplegia & $10(0.3 \%)$ & $3(0.5 \%)$ & $6(0.5)$ & $3(0.5)$ & 0.0006 \\
\hline - Renal disease & $83(2.8 \%)$ & $31(4.7 \%)$ & $42(3.3)$ & $31(4.8)$ & 0.0767 \\
\hline $\begin{array}{l}\text { - Any malignancy, including } \\
\text { lymphoma and leukemia }\end{array}$ & $2927(99.6 \%)$ & $647(98.8 \%)$ & $1289(99.6)$ & $645(98.8)$ & 0.0939 \\
\hline - Moderate or severe liver disease & $6(0.2 \%)$ & $5(0.8 \%)$ & $3(0.2)$ & $5(0.8)$ & 0.0758 \\
\hline - Metastatic solid tumor & $309(10.5 \%)$ & $82(12.5 \%)$ & $176(13.6)$ & $82(12.6)$ & 0.031 \\
\hline- HIV & $2(0.1 \%)$ & $0(0 \%)$ & $0(0)$ & $0(0)$ & NA \\
\hline
\end{tabular}




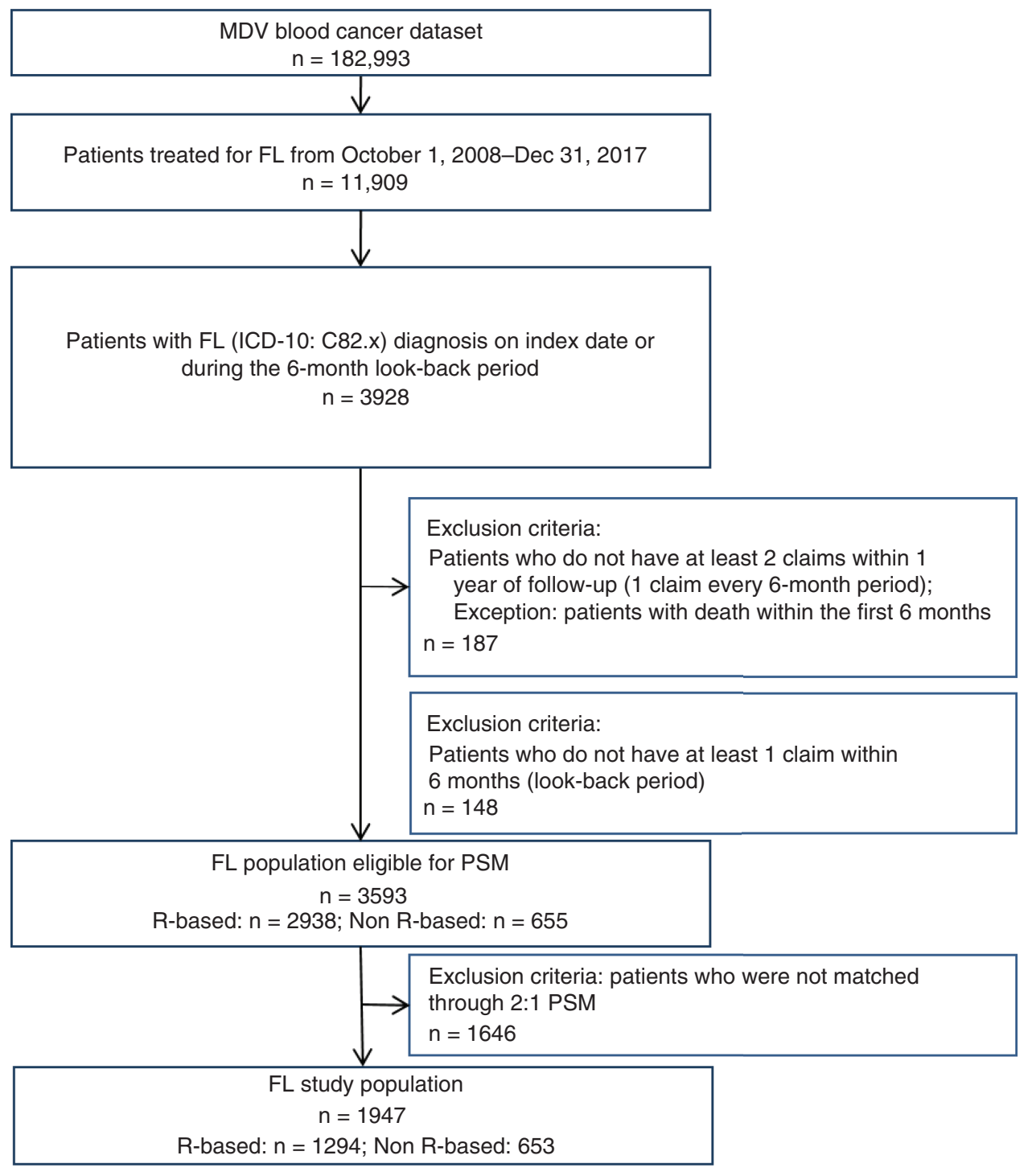

Figure 2. Patient attrition for follicular lymphoma patients.

FL: Follicular lymphoma; ICD-10: International Classification of Diseases, 10th revision; MDV: Medical Data Vision; PSM: Propensity score matching; R: Rituximab.

were mild liver disease ( 21.5 vs $20.4 \%$, respectively), chronic pulmonary disease (18.6 vs $21.0 \%)$ and congestive heart failure (16.3 vs $17.8 \%)$. Additional baseline statistics are listed in Supplementary Table 1. Most patients with comorbid metastatic solid tumors were $<66$ years old. Over $80 \%$ of the cohort identified started their index treatment on or after 2013.

\section{Treatment characterization}

Treatment patterns (Table 2) showed that of patients who initially received R-based therapy, 35.0\% switched to a non-R-based therapy as a second line (2L) of therapy. This proportion increased to $45.0 \%$ of the R-based group receiving non-R-based therapy in the third line of therapy (3L). For patients initially receiving a non-R-based regimen, $54.2 \%$ were still receiving non-R-based treatment by $2 \mathrm{~L}$ and $69.9 \%$ by $3 \mathrm{~L}$. A higher proportion of R-based regimen patients received only 1 line of therapy (46.3\%) compared with patients receiving non-R-based therapy (37.5\%). The non-R-based regimen group had a consistently larger proportion of patients receiving multiple lines of therapy, from two lines to more than six lines. Treatment progression for lines 1-3 are illustrated in Supplementary Figure 1 for those who initiated their $1 \mathrm{~L}$ before and after the approval of bendamustine, respectively. For non-Rbased regimens, the mean length of the index treatment was shorter than R-based regimens by more than 40 days 
Table 2. Treatment patterns of follicular lymphoma.

\begin{tabular}{|c|c|c|}
\hline Treatment patterns for FL $(n=1947)$ & R-based regimens $(n=1294)$ & Non-R-based regimens $(n=653)$ \\
\hline \multicolumn{3}{|l|}{ Index regimen, $\mathrm{n}(\%)$} \\
\hline - R-CHOP & $534(41.3)$ & (0) \\
\hline - R-CVP & $209(16.2)$ & (0) \\
\hline - $\mathrm{R}+$ bendamustine & $273(21.1)$ & (0) \\
\hline - $\mathrm{R}+$ other chemotherapy or dexamethasone ${ }^{\dagger}$ & $142(11.0)$ & (0) \\
\hline - R monotherapy & $136(10.5)$ & (0) \\
\hline - Other (immunotherapy, targeted therapy, hormone therapy) without R & (0) & $29(4.4)$ \\
\hline- NOS & (0) & $348(53.3)$ \\
\hline Subsequent regimen $2 \mathrm{~L}, \mathrm{n}(\%)$ & $n=695(100)$ & $\mathrm{n}=408(100)$ \\
\hline - $\mathrm{R}-\mathrm{CHOP}$ & $51(7.3)$ & $42(10.3)$ \\
\hline - R-CVP & $24(3.5)$ & $28(6.9)$ \\
\hline - Other (immunotherapy, targeted therapy, hormone therapy) without R & $8(1.2)$ & $11(2.7)$ \\
\hline- NOS & $116(16.7)$ & $111(27.2)$ \\
\hline - Subsequent regimen $3 \mathrm{~L}, \mathrm{n}(\%)$ & $\mathrm{n}=387(\mathbf{1 0 0})$ & $\mathrm{n}=237(100)$ \\
\hline$-\mathrm{R}-\mathrm{CHOP}$ & $22(5.7)$ & $6(2.5)$ \\
\hline - R-CVP & $6(1.6)$ & $4(1.7)$ \\
\hline - $\mathrm{R}+$ bendamustine & $53(13.7)$ & $37(15.6)$ \\
\hline - $\mathrm{R}+$ chemotherapy dexamethasone $\S$ & $54(14.0)$ & $21(8.9)$ \\
\hline - R monotherapy & $78(20.2)$ & $14(5.9)$ \\
\hline - Chemotherapy without $R$ & $80(20.7)$ & $83(35)$ \\
\hline - Other (immunotherapy, targeted therapy, hormone therapy) without R & $7(1.8)$ & $7(3.0)$ \\
\hline-5 & $51(3.9)$ & $36(5.5)$ \\
\hline$-6+$ & $82(6.3)$ & $50(7.7)$ \\
\hline \multicolumn{3}{|l|}{ Duration of index regimen (days) } \\
\hline - Mean (SD) & $168.8(197.3)$ & $121.2(288.7)$ \\
\hline - Median (Q1, Q3) & $128.0(83.0,169.0)$ & $33.0(8.0,104.0)$ \\
\hline - Minimum, maximum & $1.0,3058.0$ & $1.0,2757.0$ \\
\hline Duration of $2 \mathrm{~L}$ regimen (days) & $\mathrm{n}=695(100)$ & $\mathrm{n}=408(100)$ \\
\hline - Mean (SD) & $119.1(182.5)$ & $130.7(205.4)$ \\
\hline - Median (Q1, Q3) & $59.0(22.0,141.0)$ & $72.0(26.0,142.0)$ \\
\hline - Minimum, maximum & $1.0,1583.0$ & $1.0,1493.0$ \\
\hline Duration of $3 \mathrm{~L}$ regimen (days) & $\mathrm{n}=387(100)$ & $\mathrm{n}=237(100)$ \\
\hline Mean (SD) & $97.2(189.7)$ & $122.7(232.0)$ \\
\hline - Median (Q1, Q3) & $40.0(9.0,109.0)$ & $55.0(8.0,126.0)$ \\
\hline - Minimum, maximum & $1.0,1889.0$ & $1.0,1676.0$ \\
\hline Patients using $\mathrm{R}$ maintenance therapy after $1 \mathrm{~L}^{\dagger}, \mathrm{n}(\%)$ & $85(7.3)$ & 0 \\
\hline \multicolumn{3}{|c|}{$\begin{array}{l}\dagger \text { † Maintenance therapy was explored in patients with R-based 1L regimens who continued therapy with R after } 1 \mathrm{~L} \text { ended. } \\
\text { 1L: First line; 2L: Second line; 3L: Third line; 4L: Fourth line; 5L: Fifth line; 6L: Sixth line; CHOP: Cyclophosphamide, doxorubicin, vincristine and prednisone; CVP: Cyclophosphamide } \\
\text { vincristine and prednisone; FL: Follicular lymphoma; Q1, Q3: 1st and 3rd quartile; NOS: Not otherwise specified; R: Rituximab; R-CHOP: Rituximab plus cyclophosphamide, doxorubicir } \\
\text { vincristine and prednisone; R-CVP: Rituximab plus cyclophosphamide, vincristine and prednisone; SD: Standard deviation. }\end{array}$} \\
\hline
\end{tabular}


Table 2. Treatment patterns of follicular lymphoma (cont.).

\begin{tabular}{|c|c|c|}
\hline Treatment patterns for FL $(n=1947)$ & $R$-based regimens $(n=1294)$ & Non-R-based regimens $(n=653)$ \\
\hline \multicolumn{3}{|l|}{ Patients with stem cell transplant, $\mathrm{n}(\%)$} \\
\hline - During index regimen (prior to $2 \mathrm{~L}$ ) & $1(0.1)$ & $2(0.3)$ \\
\hline - During $2 \mathrm{~L}$ (prior to $3 \mathrm{~L}$ ) & $8(0.6)$ & $1(0.2)$ \\
\hline - During $3 \mathrm{~L}$ (prior to $4 \mathrm{~L}$ ) & $9(0.7)$ & $3(0.5)$ \\
\hline - During $4 \mathrm{~L}$ (prior to $5 \mathrm{~L}$ ) & $7(0.5)$ & $0(0)$ \\
\hline - During $5 \mathrm{~L}$ (prior to $6 \mathrm{~L}+$ ) & $5(0.4)$ & $1(0.2)$ \\
\hline \multicolumn{3}{|l|}{ Patients with radiation therapy, $\mathrm{n}(\%)$} \\
\hline - During index regimen or within 30 days prior to initiation & $64(4.9)$ & $33(5.1)$ \\
\hline - During entire patient follow-up period & $127(9.8)$ & $74(11.3)$ \\
\hline \multicolumn{3}{|c|}{$\begin{array}{l}\dagger \text { Maintenance therapy was explored in patients with R-based } 1 \mathrm{~L} \text { regimens who continued therapy with R after } 1 \mathrm{~L} \text { ended. } \\
\text { 1L: First line; } 2 \mathrm{~L} \text { : Second line; 3L: Third line; } 4 \mathrm{~L} \text { : Fourth line; } 5 \mathrm{~L} \text { : Fifth line; } 6 \mathrm{~L} \text { : Sixth line; CHOP: Cyclophosphamide, doxorubicin, vincristine and prednisone; CVP: Cyclophosphamid } \\
\text { vincristine and prednisone; FL: Follicular lymphoma; Q1, Q3: 1st and 3rd quartile; NOS: Not otherwise specified; R: Rituximab; R-CHOP: Rituximab plus cyclophosphamide, doxorubici } \\
\text { vincristine and prednisone; R-CVP: Rituximab plus cyclophosphamide, vincristine and prednisone; SD: Standard deviation. }\end{array}$} \\
\hline
\end{tabular}

Table 3. Healthcare resource utilization during the follow-up period, overall.

\begin{tabular}{|c|c|c|c|c|c|c|c|}
\hline HCRU outcome & $\mathrm{n}$ & $\begin{array}{l}\text { All regimens } \\
\text { Mean (SD) }\end{array}$ & \multicolumn{2}{|c|}{$\begin{array}{l}\text { R-based regimens } \\
\quad(n=1294)\end{array}$} & \multicolumn{2}{|c|}{$\begin{array}{l}\text { Non- } \mathrm{R} \text {-based regimens } \\
\qquad(\mathrm{n}=653)\end{array}$} & p-value \\
\hline Outpatient visits (n) & 1899 & 49.7 (36.1) & 1283 & $51.2(35.1)$ & 616 & $46.8(38.1)$ & 0.0168 \\
\hline LOS $^{\dagger}$ days overall & 1819 & 74.5 (83.9) & 1264 & $70.9(78.5)$ & 555 & $82.8(94.6)$ & 0.0096 \\
\hline ICU admissions ( $n$ ) & 43 & $1.0(0.2)$ & 23 & $1.1(0.3)$ & 20 & $1.0(0.0)$ & 0.1619 \\
\hline $\begin{array}{l}\text { Radiation therapy procedures during } \\
\text { follow-up }(n)\end{array}$ & 201 & $20.0(12.3)$ & 127 & $20.5(12.7)$ & 74 & $19.1(11.7)$ & 0.4635 \\
\hline
\end{tabular}

$\dagger$ Among hospitalized patients.

ER: Emergency room; HCRU: Healthcare resource utilization; ICU: Intensive care unit; LOS: Length of stay; NA: Not applicable; R: Rituximab; SD: Standard deviation.

(168.8 vs 121.2 days). The inverse trend was observed in lines 2 and 3, where patients with R-based index regimens had a shorter duration by more than 10 days. Slightly more patients receiving non-R-based regimens for FL also underwent radiation therapy compared with those receiving R-based treatment over the entire patient follow-up period (11.3 vs $9.8 \%)$.

\section{Healthcare resource utilization \& costs}

HCRU during the follow-up period is shown in Table 3. Overall, the mean (SD) number of outpatient visits was 49.7 (36.1). Patients receiving R-based therapy had more outpatient visits compared with non-R-based therapy (51.2 vs 46.8). Mean number of hospitalizations was the same (3.8) for the entire FL patient group when stratified by R-based regimen. The mean length of stay for these hospitalizations was longer for patients receiving non-Rbased therapy (82.8 days) compared with patients treated with R-based regimens (70.9 days). There was very little difference in the number of ICU or emergency room visits as well as the number of SCTs or radiation treatments performed regardless of R-based treatment status. Detailed HCRU by each year of follow-up are additionally reported in Supplementary Table 2.

Direct adjusted costs during the follow-up period are shown by overall and yearly costs in Table 4. The mean (SD) of total medical costs over the entire follow-up period was $\$ 66,247.41(\$ 51,950.18)$ and was significantly higher for R-based regimens compared with non-R-based regimens $(\$ 69,601.68$ vs 59,600.51; p < 0.0001). During the first year of follow-up the total mean (SD) costs for all regimens were $\$ 39,481.78(\$ 27,109.82)$. When stratified by treatment regimen type, mean first year costs were $\$ 43,407.60$ for R-based regimens and $\$ 31,702.27$ for non-R-based regimens ( $\mathrm{p}<0.0001)$. However, from the second year of follow-up, R-based regimens had lower 


\begin{tabular}{|c|c|c|c|c|c|c|c|}
\hline \multirow[t]{2}{*}{ Cost outcome } & \multicolumn{2}{|r|}{ All regimens } & \multicolumn{2}{|c|}{$\mathbf{R}$-based regimens } & \multicolumn{2}{|c|}{ Non-R-based regimens } & \multirow[t]{2}{*}{ p-value } \\
\hline & $\mathrm{n}$ & Mean (SD) & n & Mean (SD) & $\mathbf{n}$ & Mean (SD) & \\
\hline Total costs & 1947 & $66247.41(51950.18)$ & 1294 & 69601.68 (47772.04) & 653 & $59600.51(58844.37)$ & $<0.0001$ \\
\hline - During year 1 & 1947 & $39481.78(27109.82)$ & 1294 & $43407.60(24498.76)$ & 653 & 31702.27 (30201.01) & $<0.0001$ \\
\hline - During year 2 & 1749 & $11479.23(18257.54)$ & 1204 & $10673.01(16803.02)$ & 545 & $13260.31(21026.33)$ & 0.0011 \\
\hline - During year 3 & 1451 & 9942.44 (17699.58) & 1007 & 9343.04 (15954.74) & 444 & $11301.88(21087.02)$ & 0.0120 \\
\hline - During year 4 & 1091 & 7656.07 (14563.08) & 774 & 7037.73 (13689.12) & 317 & 9165.85 (16429.58) & 0.0049 \\
\hline - During year 5 & 693 & $7735.84(16743.66)$ & 484 & 7378.23 (16410.84) & 209 & 8563.99 (17502.42) & 0.2295 \\
\hline Inpatient cost, total & 1819 & $40641.86(43082.93)$ & 1264 & 38761.14 (41087.79) & 555 & $44925.14(47073.23)$ & 0.0027 \\
\hline - During year 1 & 1732 & $27716.82(28096.16)$ & 1237 & $26647.76(27250.16)$ & 495 & 30388.36 (29968.82) & 0.0078 \\
\hline - During year 2 & 486 & $20257.99(23687.21)$ & 287 & $19563.60(22567.20)$ & 199 & $21259.45(25239.66)$ & 0.3781 \\
\hline - During year 3 & 355 & $19747.00(23879.19)$ & 221 & $18953.22(21052.81)$ & 134 & $21056.15(27960.62)$ & 0.3422 \\
\hline - During year 4 & 234 & $19183.11(19246.13)$ & 153 & $19589.32(18869.24)$ & 81 & $18415.80(20035.62)$ & 0.6424 \\
\hline - During year 5 & 139 & $19725.05(22044.04)$ & 97 & $20034.39(23271.70)$ & 42 & 19010.62 (19151.56) & 0.7864 \\
\hline ICU cost, total & 43 & 3272.53 (3694.49) & 23 & 3633.46 (3924.72) & 20 & $2857.46(3463.74)$ & 0.3892 \\
\hline - During year 1 & 27 & $3729.72(4165.77)$ & 13 & $4318.08(4336.26)$ & 14 & $3183.38(4084.47)$ & 0.3895 \\
\hline - During year 2 & 4 & $1958.62(1793.21)$ & 4 & $1958.62(1793.21)$ & $\mathrm{N} / \mathrm{A}$ & $\mathrm{N} / \mathrm{A}$ & $\mathrm{N} / \mathrm{A}$ \\
\hline - During year 3 & 3 & $1786.71(638.92)$ & $\mathrm{N} / \mathrm{A}$ & NA & 3 & $1786.71(638.92)$ & $\mathrm{N} / \mathrm{A}$ \\
\hline - During year 4 & 4 & $4268.72(4018.84)$ & 4 & $4268.72(4018.84)$ & $\mathrm{N} / \mathrm{A}$ & $\mathrm{N} / \mathrm{A}$ & $\mathrm{N} / \mathrm{A}$ \\
\hline - During year 5 & 4 & $1614.87(1546.43)$ & 2 & $831.93(0.00)$ & 2 & $2397.80(2173.11)$ & 0.0851 \\
\hline Outpatient cost, total & 1899 & $28992.19(27440.65)$ & 1283 & 32011.29 (25609.92) & 616 & 22704.02 (29967.44) & $<0.0001$ \\
\hline - During year 1 & 1897 & $15216.39(12610.15)$ & 1282 & 18101.52 (12428.92) & 615 & 9202.19 (10729.98) & $<0.0001$ \\
\hline - During year 2 & 1724 & $5934.91(8252.08)$ & 1192 & $6070.09(8134.45)$ & 532 & $5632.03(8509.75)$ & 0.2111 \\
\hline - During year 3 & 1439 & $5153.78(8156.46)$ & 1000 & $5219.78(7962.84)$ & 439 & $5003.44(8588.70)$ & 0.5316 \\
\hline - During year 4 & 1082 & $3571.10(7033.20)$ & 768 & $3190.15(5827.06)$ & 314 & $4502.85(9295.53)$ & $<0.0001$ \\
\hline - During year 5 & 689 & $3801.39(9712.27)$ & 481 & $3384.05(8237.68)$ & 208 & 4766.48 (12442.69) & 0.0015 \\
\hline Cancer treatment costs, total & 1947 & 28140.88 (25996.49) & 1294 & $32550.86(23327.81)$ & 653 & $19401.96(28684.68)$ & $<0.0001$ \\
\hline - During year 1 & 1947 & $18534.91(14093.38)$ & 1294 & $22242.60(12025.52)$ & 653 & 11187.66 (14989.65) & $<0.0001$ \\
\hline - During year 2 & 690 & 11086.66 (11477.29) & 435 & $12667.05(10838.92)$ & 255 & $8390.70(12042.18)$ & 0.0007 \\
\hline - During year 3 & 531 & $10088.82(11479.65)$ & 350 & $11213.96(11106.71)$ & 181 & 7913.15 (11900.64) & 0.0131 \\
\hline - During year 4 & 281 & $9158.41(11033.03)$ & 175 & $9916.25(9912.92)$ & 106 & $7907.25(12618.61)$ & 0.2441 \\
\hline - During year 5 & 157 & $11094.19(14857.14)$ & 98 & $12470.84(15204.10)$ & 59 & 8807.54 (14091.68) & 0.2036 \\
\hline Other pharmacy costs, total & 1947 & $11037.70(17232.02)$ & 1294 & $10432.08(15521.03)$ & 653 & $12237.80(20158.44)$ & 0.0043 \\
\hline - During year 1 & 1946 & $5835.15(9176.37)$ & 1294 & $5809.53(8151.29)$ & 652 & $5885.98(10936.65)$ & 0.8173 \\
\hline - During year 2 & 1622 & $2355.98(6022.64)$ & 1115 & $1975.70(5219.14)$ & 507 & $3192.30(7430.39)$ & $<0.0001$ \\
\hline - During year 3 & 1299 & $2163.90(6157.91)$ & 899 & $1810.86(5028.44)$ & 400 & $2957.36(8095.59)$ & $<0.0001$ \\
\hline - During year 4 & 904 & $1886.86(4985.90)$ & 628 & $1641.09(4624.90)$ & 276 & $2446.08(5692.84)$ & 0.0003 \\
\hline - During year 5 & 575 & $1997.70(6073.57)$ & 394 & $1783.58(5599.27)$ & 181 & $2463.78(6990.15)$ & 0.0279 \\
\hline Cost of stem cell transplants & 37 & $3473.29(1306.75)$ & 30 & $3404.61(1275.98)$ & 7 & $3767.67(1500.49)$ & 0.4442 \\
\hline Cost of radiation therapy procedures & 201 & $3132.88(2142.77)$ & 127 & $3195.95(2229.50)$ & 74 & $3024.63(1995.26)$ & 0.5641 \\
\hline
\end{tabular}

total costs compared with non-R-based regimens $(\$ 10,673.01$ vs $13,260.31 ; \mathrm{p}=0.0011)$. This trend continued, with statistically significant differences, until the 5th year of follow-up ( $\$ 7378.23$ vs $8563.99 ; \mathrm{p}=0.2295$ ).

Similarly, overall mean costs for R-based regimens were higher for ICU compared with non-R-based regimens (\$3633.46 vs 2857.46$)$, outpatient $(\$ 32,011.29$ vs $22,704.02)$ and cancer treatment $(\$ 32,550.86$ vs $19,401.96)$ across all years. Cancer treatment costs were consistently lower for non-R-based therapy in each year of follow-up, while outpatient costs became significantly lower for R-based regimens compared with non-R-based therapy in the fourth $(\$ 3190.15$ vs 4502.85 ; $\mathrm{p}<0.0001)$ and fifth year $(\$ 3384.05$ vs $4766.48 ; \mathrm{p}=0.0015)$. In contrast, the overall inpatient costs accrued with R-based regimens were lower than non-R-based $(\$ 38,761.14$ vs $44,925.14)$ $(\mathrm{p}=0.0027)$. The lower inpatient costs of R-based regimens remained constant for each of the 5 years of follow- 
Table 5. Overall survival and time-to-next-treatment outcomes.

\begin{tabular}{|c|c|c|c|}
\hline Characteristics & All regimens $(n=1947)$ & $R$-based regimens $(n=1284)$ & Non-R-based regimens $(n=653)$ \\
\hline Deaths (n [\%]) & $270(13.9 \%)$ & $126(9.7 \%)$ & $144(22.1 \%)$ \\
\hline \multicolumn{4}{|l|}{ KM OS estimate } \\
\hline - Median $(95 \% \mathrm{Cl})$ & Not reached & Not reached & Not reached \\
\hline Number of patients with next treatment (n [\%]) & $1103(56.7 \%)$ & $695(53.7 \%)$ & $408(62.5 \%)$ \\
\hline $\begin{array}{l}\text { Number of patients with next treatment or death ( } \\
{[\%] \text { ) }}\end{array}$ & $1161(59.6 \%)$ & $709(54.8 \%)$ & $452(69.2 \%)$ \\
\hline \multicolumn{4}{|l|}{ TTNT estimate, death as competing risk } \\
\hline - $\mathrm{HR}(95 \% \mathrm{Cl})$ & & $0.702(0.620,0.796)$ & Ref \\
\hline
\end{tabular}

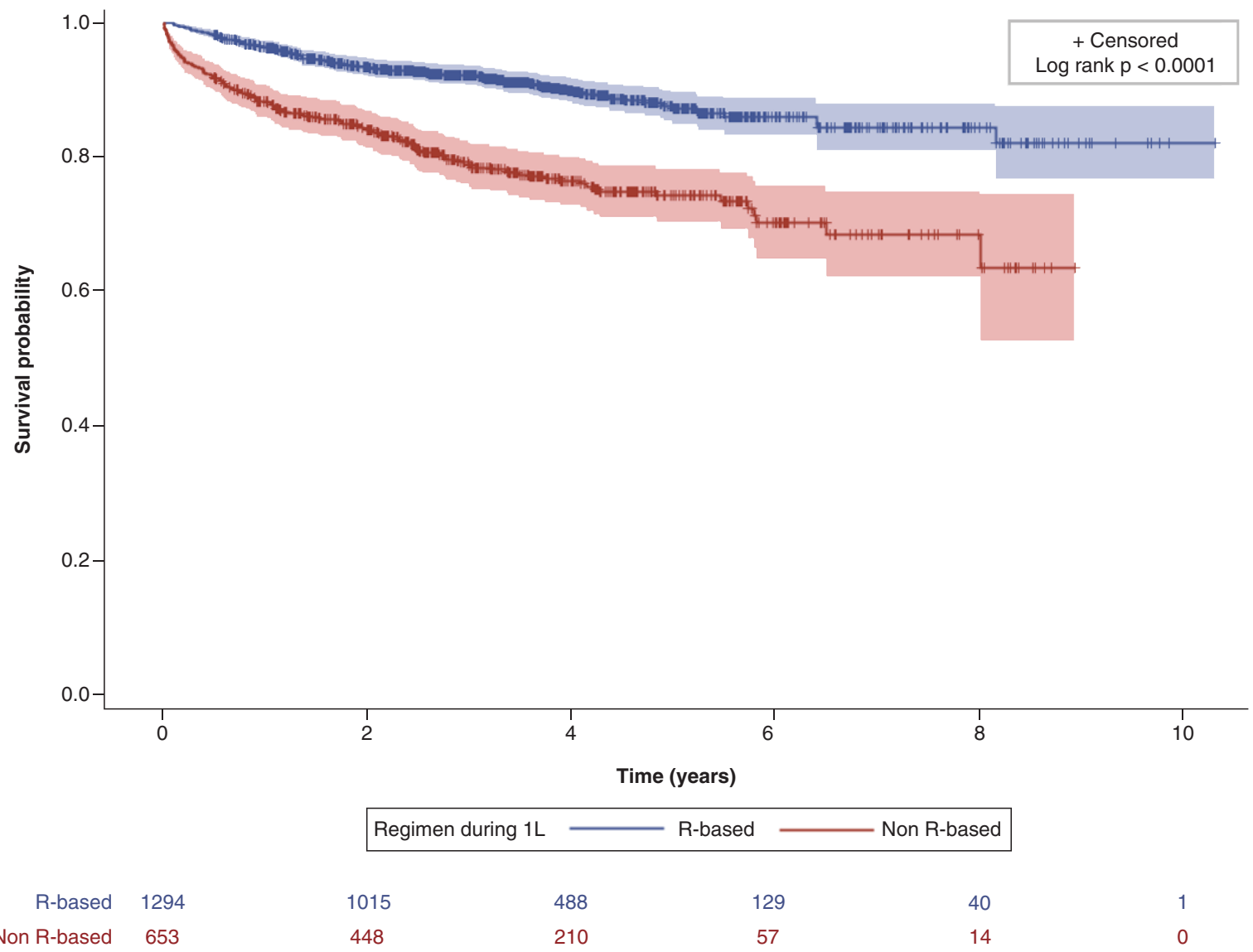

Figure 3. Kaplan-Meier plot for overall survival stratified by R-based and non R-based regimen.

1L: First line; R: Rituximab.

up. Both the overall and yearly costs for other pharmacy costs were also consistently lower in R-based regimens compared with non-R-based regimens (overall average cost: $\$ 10,432.08$ vs $12,237.80)(p=0.0043$ ). The costs of SCTs and radiation therapy procedures during follow-up were similar between the treatment groups. The nominal costs prior to adjusting for inflation are also presented in Supplementary Table 3.

Overall survival

Overall, there were 270 deaths (13.9\% of patients) over the analysis period for the entire group of patients with FL. Among patients receiving non-R-based regimens for FL, mortality was roughly double that of R-based patients (22.1 vs 9.7\%, respectively) (Table 5). Based on the OS KM curve, R-based regimens had significantly better survival ( $\mathrm{p}<0.0001$ ), although a median was not reached in either group (Figure 3). A significantly better OS was 

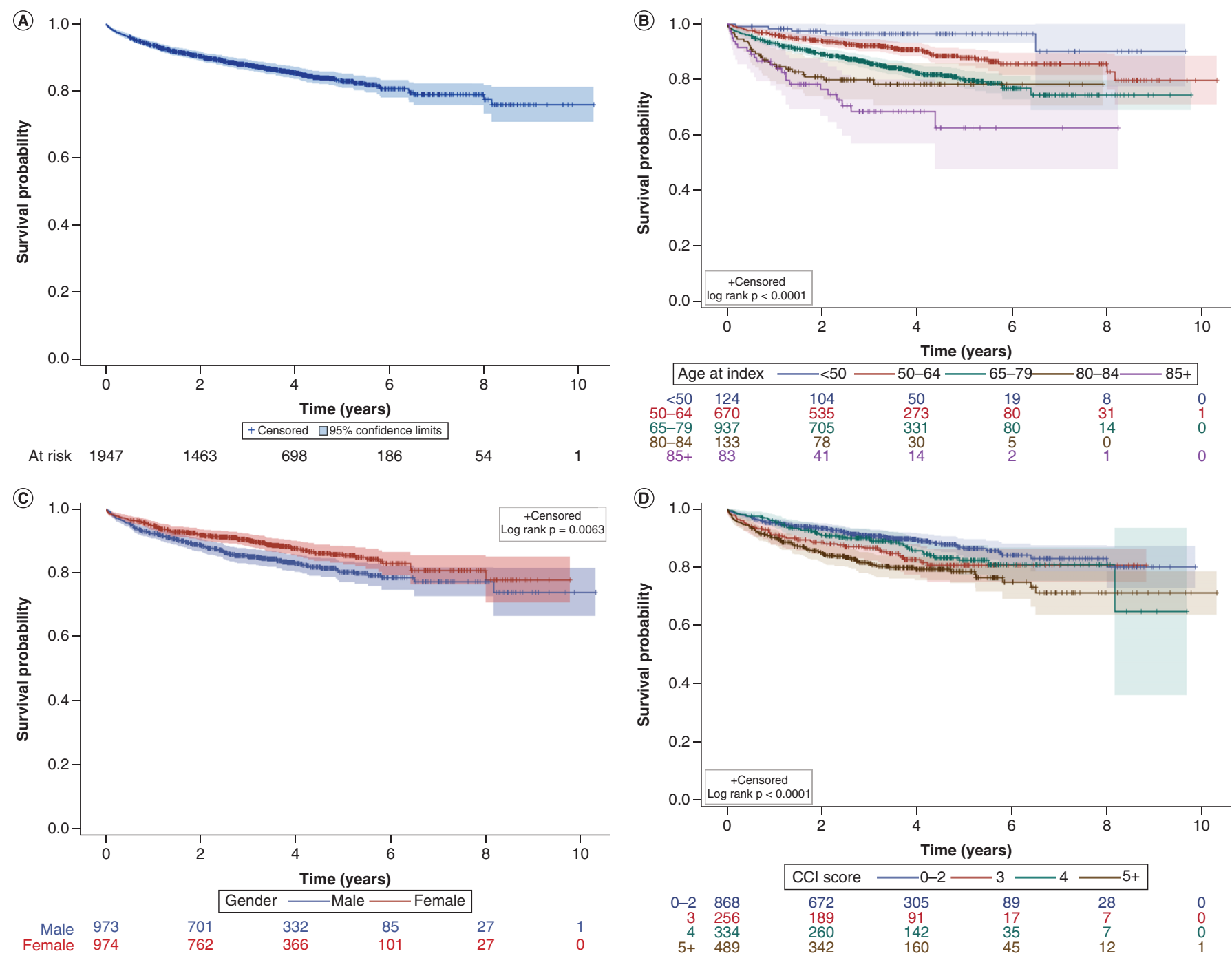

Figure 4. Kaplan-Meier plot for overall survival stratified by patient baseline attributes. Kaplan-Meier plot for overall survival for (A) all follicular lymphoma patients and stratified by (B) age group, (C) sex, (D) CCI score.

CCl: Charlson comorbidity index.

also found with lower age, female and lower CCI score (Figure 4). Detailed summary statistics on OS are reported in Supplementary Table 4.

\section{Time to next treatment}

There were a total 1103 patients (56.7\%) who progressed to subsequent lines of therapy and 1161 (59.6\%) who either progressed in treatment or died (Table 5). Patients who received R-based therapy during 1L marked a significant reduction in risk of progressing to subsequent therapy compared with those who received non-R-based therapy (HR: 0.702; 95\% CI: 0.620-0.796). The cumulative incidence function plot stratified by treatment group is shown in Figure 5. The risk of treatment progression was also significantly reduced in younger age groups (Figure 6), although no differences were observed in terms of sex and CCI score. Detailed summary statistics on TTNT can also be found in Supplementary Table 4.

\section{Discussion}

To our knowledge, this is the first study to comprehensively evaluate treatment patterns, cost, HCRU and clinical outcomes among patients with FL in Japan using a propensity score-matched cohort. The identified baseline demographics (i.e., age and sex) were comparable to FL patients characterized by Morrison et al. in the USA [9]. 


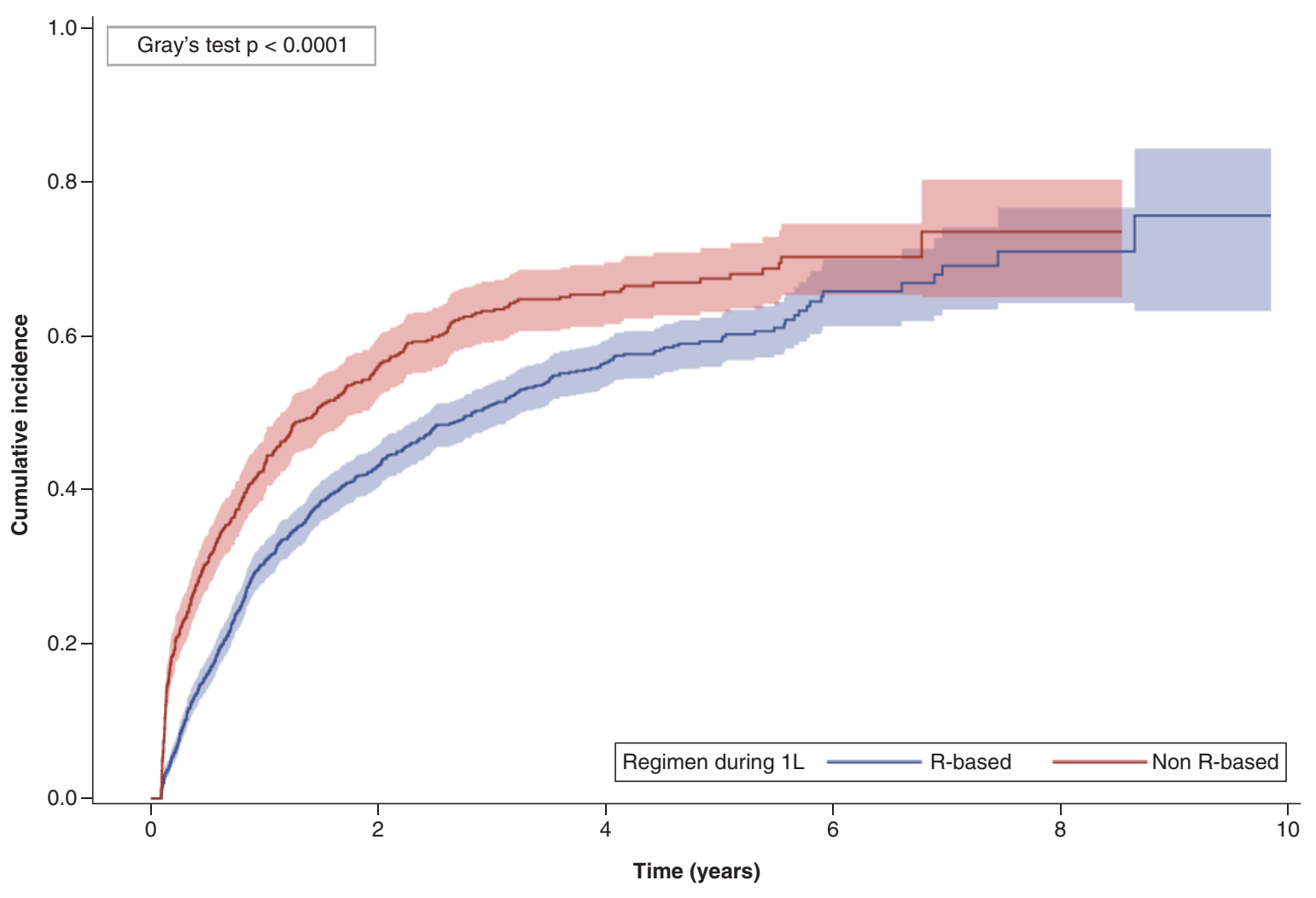

Figure 5. Cumulative incidence function plot for time-to-next-treatment stratified by $R$-based regimen and non R-based regimen.

1L: First-line; R: Rituximab.

For FL patients who were eligible for PSM in the present analysis, about two-thirds of patients received an R-based regimen. This is a distribution that is similar to prior real-world evidence of FL treatment patterns, suggesting that the regimens identified reflect FL treatment. In terms of comorbidities, less than $1 \%$ of patients had a CCI score of 0 and almost half of both treatment groups had a CCI of 2. Over half of patients had a CCI score of 3 or higher. Interpretation of CCI score distribution could be explained by our patient selection definition, which required them to have a diagnosis of any malignancy (part of the CCI definition) on or prior to their index date, thus the diagnosis of FL itself was included in the CCI. Approximately a fifth of the patients had the following commonly observed comorbidities: chronic pulmonary disease, mild liver disease and congestive heart failure. Physicians report that they take comorbid conditions into account when deciding NHL treatment intensity but evidence remains poor and heterogeneous about the comorbidities and outcomes in FL [18]. It is possible that the utilization of R-based regimens is affected depending on the treating physicians' experience with comorbidities and FL.

According to the clinical guidelines, chemo-immunotherapy is the recommended treatment for FL patients across all stages for patients who are not otherwise 'watch-and-wait' or radiation therapy recipients. These results are mostly consistent with the Japanese Society of Hematology guidelines [19], with chemo-immunotherapy observed in $72.4 \%$ of patients and $\mathrm{R}$ alone in $9.3 \%$ of patients.

Notably, patients on R-based regimens had a significantly higher number of outpatient visits compared with therapies without $\mathrm{R}$ due to the possibility that patients might be receiving $\mathrm{R}$-maintenance therapy, although the direction of effect was reversed on and after year 2 . The $7 \%$ of patients on R-based regimens with $\mathrm{R}$ maintenance therapy after their index treatment could potentially contribute to the high number of outpatient visits during the first year. Patients on R-based regimens also marked a significantly lower overall length of stay and number of hospitalizations in year 1. However, overall number of hospitalizations, ER visits and ICU visits were comparable between the groups.

Total costs for FL patients in Japan in this study were found to vary widely, with a mean (SD) of $\$ 66,247.41$ $(\$ 51,950.18)$ USD. The heterogeneity of these costs may reflect the heterogeneity inherent in the FL patient 
(A)

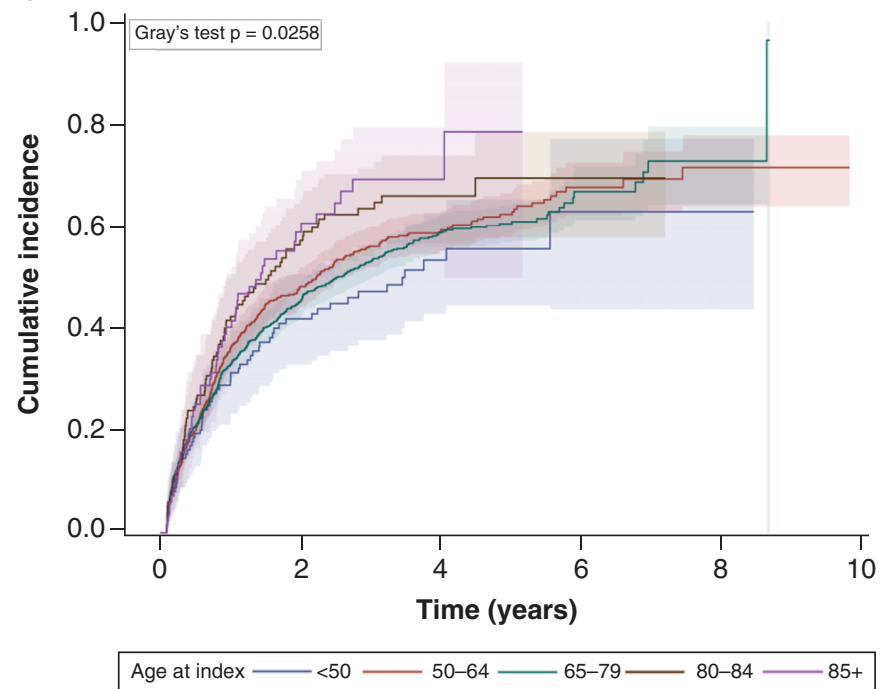

(B)

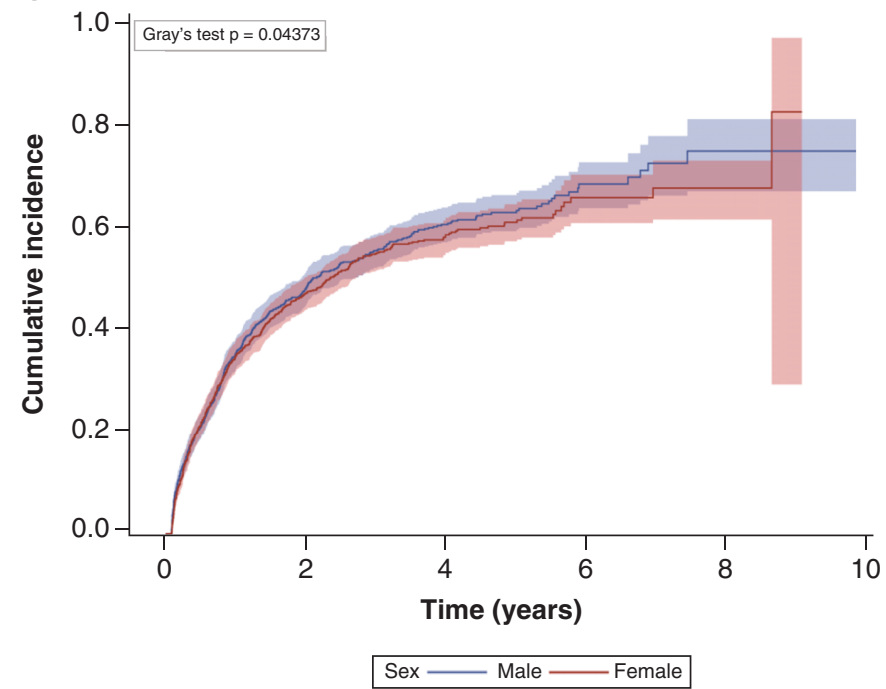

(C)

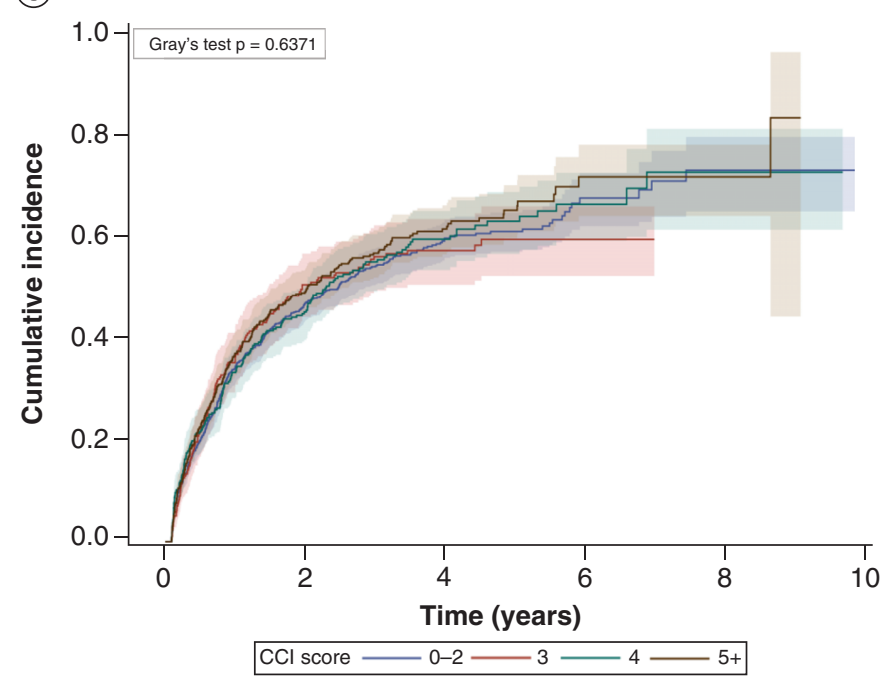

Figure 6. Cumulative incidence function plot for time-to-next treatment stratified by patient baseline characteristics. Cumulative incidence function plot for time-to-next-treatment stratified by (A) age group, (B) sex, (C) CCl score. CCl: Charlson comorbidity index.

population itself. Patients with R-based 1L regimens had significantly higher overall medical costs but we observed a lower yearly cost on and after year 3 . The first-year costs found in this study $(\$ 39,481.78)$ was similar to that was reported by another US study, which found the first-year economic burden in FL patients to be $\$ 36,000$ [20]. Patients with R-based 1L regimens had significantly higher outpatient costs, but we observed a reversed direction of effect in years 4. Longer treatment may significantly increase healthcare expenditure, as $\mathrm{R}$ is priced at JPY 22,553 ( $\$ 210.80$ USD) per $100 \mathrm{mg}$ vial [21]. Additionally, R-based regimens require multiple days of injections, which may contribute to the higher outpatient utilization for patients on R-based therapies.

Although the cancer pharmacy and outpatient costs were higher for R-based regimens, the inpatient costs were significantly lower. These findings show the high costs of outpatient and pharmacy burden driven by R; however, it has also shown the potential effect of longer-term benefits. A retrospective study of the National Health Service (UK) in 2018 also found a wide range of costs and survival outcomes [22] and recommended future cost modeling based on a lifetime horizon approach, as results from restricted time horizons may not be appropriate in all scenarios. 
In previous studies [22-25], R-based therapies have shown to be relatively cost-effective options compared with non- $R$ therapy in the use of $\mathrm{R}$ in induction monotherapy, combination with chemotherapy and as maintenance therapy.

The FL patients identified in this study generally had long survival, with only $13.9 \%$ who died during the follow-up period, or a presumed survival rate of $86.1 \%$. These results are very similar to the findings in another Japanese study of FL patients who showed 5-year OS rates ranging from 84.4 to $93.5 \%$ [26]. These results were cited in a systematic review by Monga et al. [27], who described a 5-year OS range from 48.9 to $93.5 \%$ in the most elderly population as well. In this study, OS findings were stratified, further characterizing this patient population.

More specifically, stratified our OS KM survival analyses showed worse survival with increasing age, male sex and chemotherapy regimens without R. Although all other groups had similar survival curves, in contrast, R-based therapies performed better than regimens in the chemotherapy without $\mathrm{R}$ group. In this study, slightly higher OS was observed compared with the 2019 US database study by Morrison et al. (90.6\% 3-year OS vs 86.9\% 2-year survival) $[9,28]$.

Our TTNT results were in favorable agreement in trend with the above OS analysis results. As patients who have progressive disease were found to have significantly higher frequencies of outpatient visits, laboratory procedures, acute care visits and intensive chemotherapy [29], it is paramount that $1 \mathrm{~L}$ therapy is effective to delay disease progression and thus, minimize HCRU and direct costs.

The findings herein should be interpreted in light of several limitations. First, the MDV database does not indicate the disease treated with each drug claim, therefore treatment groupings were made in consultation with the clinical experts based on the relevance from clinical plausibility perspective. The complete treatment history of the included patients is not recorded in this database, therefore their $1 \mathrm{~L}$ regimen may or may not be their true first line of therapy. Analysis was based on a patient's first treatment during the identification period and required at least 6 months of look-back with a FL diagnosis. An assumption was made that most patients were treatment naive and the distribution of the $1 \mathrm{~L}$ regimens was consistent with the prior literature. Also, 'watch and wait' was difficult to define during this study, therefore we did not include it as part of the scope of this study. Further, mortality data were captured only through patient's hospital discharge records, limiting the robustness of OS calculations.

In our study, we observed no impact of obinutuzumab (Gazyva), approved for naive and relapsed/refractory FL in Japan in July 2018, due to our data study period (2008-2017) occurring prior to its approval. We also observed no impact of the R subcutaneous injection formulation. In Western countries, the subcutaneous injection formulation of R, especially used in maintenance therapy, was approved in the USA in June 2017. However, it has not been approved in Japan yet. Several R biosimilar drugs have been launched in Japan for B cell NHL in recent years. R biosimilar obtained approval in September 2017, but was not available as a treatment option in real-world practice until January 2018. Similarly, R biosimilar approval in Japan was obtained in September 2019 following US approval in July 2019. These treatment options were not included in the scope of our study due to the data available during the study period.

Although it was not possible to fully capture all cases of mortality, the randomness inherent in this variable should make the comparison between groups still clinically plausible.

\section{Conclusion \& future perspective}

This study revealed that patients with FL in Japan typically experienced multiple lines of therapy with diverse treatment patterns due to little consensus of FL treatment and had relatively long survival outcomes. In general, our findings report the mean (SD) total medical cost during first year was significantly higher for R-based regimens but the trend reversed on and after year 2 .

Clinical outcomes, measured by OS and TTNT, were better in patients with R-based therapies, in order of decreasing age and female. Our results underscore the superiority of R-based therapy over other regimens as the $1 \mathrm{~L}$ treatment option in balanced consideration from comparative effectiveness perspectives of clinical outcomes, with regard to mid to long-term economic burden of FL care. 


\section{Summary points}

- Newly diagnosed follicular lymphoma patients who were on rituximab (R)-based treatment had more outpatient visits compared with patients on non-R-based treatment (51.2 vs 46.8).

- The mean length of stay for hospitalizations was longer for patients receiving non-R-based therapy (82.8) compared with patients treated with R-based regimens (70.9).

- The mean (standard deviation) total medical costs were significantly higher for R-based regimens compared with non-R-based regimens ( $\$ 69,601.68$ vs $59,600.51)$.

- The mean (standard deviation) total medical cost during first year was also significantly higher for R-based regimens $(\$ 43,407.60$ vs $31,702.27)$ but the trend reversed on and after year 2 .

- Cancer treatment costs were consistently lower for non-R-based therapy in each year of follow-up, while outpatient costs became significantly higher for non-R-based therapy in years 4 and 5.

- In contrast, the overall inpatient costs accrued with R-based regimens were lower than with non-R-based regimens $(\$ 38,761.14$ vs $44,925.14)$.

- Overall costs for ICU (\$3633.46 vs 2857.46$)$, outpatient $(\$ 32,011.29$ vs $22,704.02)$ and cancer treatment $(\$ 32,550.86$ vs 19,401.96) were lower for non-R-based therapy compared with R-based therapy.

- Stratified Kaplan-Meier survival analyses showed worse survival with increasing age, male sex and chemotherapy regimens without $R$.

- Patients who received $\mathrm{R}$-based therapy during $1 \mathrm{~L}$ marked a significant reduction in the risk of progressing to subsequent therapy compared with those who received a non-R-based therapy.

- As the treatment of follicular lymphoma continues to evolve with upcoming innovative therapies, the economic impact and healthcare resource utilization need to be further investigated in these populations who receive $R$, non-R and other types of innovative therapies.

Financial \& competing interests disclosure

S Tsutsué is current employee of Celgene KK. B Crawford and J Yi are current employee of Syneos Health Japan. K Tobinai served as a consultant for Mundi Pharma, Takeda Pharmaceutaical, Celgene, Ono pharmaceutical, Janssen Pharmaceutical, HUYA Bioscience, Zenyaku Kogyo, Daiichi Sankyo, Eizai, Chugai Pharmaceutical, outside of the submitted work. K Tobinai received honaria from Solasia, Mundi Pharma, Takeda Pharmaceutaical, Ono pharmaceutical, Yakult, Meiji Seika, Janssen Pharmaceutical, BMS, HUYA Bioscience, Zenyaku Kogyo, DS, Eizai, Chugai Pharmaceutical, Verastem, Kyowa Kirin, outside of the submitted work. K Tobinai received research funding from Mundi Pharma, Takeda Pharmaceutaical, Celgene, Ono pharmaceutical, Yakult, Janssen Pharmaceutical, Eizai, Chugai Pharmaceutical, outside of the submitted work. The authors have no other relevant affiliations or financial involvement with any organization or entity with a financial interest in or financial conflict with the subject matter or materials discussed in the manuscript apart from those disclosed.

No writing assistance was utilized in the production of this manuscript.

Open access

This work is licensed under the Attribution-NonCommercial-NoDerivatives 4.0 Unported License. To view a copy of this license, visit http://creativecommons.org/licenses/by-nc-nd/4.0/

\section{References}

Papers of special note have been highlighted as: $\bullet$ of interest; $\bullet \bullet$ of considerable interest

1. PDQ Adult Treatment Editorial Board. Adult non-Hodgkin lymphoma treatment (PDQ®): health professional version. (2002). https://pubmed.ncbi.nlm.nih.gov/26389492/

2. Chihara D, Ito H, Matsuda $\mathrm{T}$ et al. Differences in incidence and trends of haematological malignancies in Japan and the United States. Br. J. Haematol. 164(4), 536-545 (2014).

3. Tsutsué S, Tobinai K, Yi J, Crawford B. Nationwide claims database analysis of treatment patterns, costs and survival of Japanese patients with diffuse large B-cell lymphoma. PLoS ONE 15(8), e0237509 (2020).

-• Reports treatment patterns and cost analysis of diffuse large B-cell lymphoma using nationwide retrospective claims database in Japan.

4. Krysiak K, Gomez F, White BS et al. Recurrent somatic mutations affecting B-cell receptor signaling pathway genes in follicular lymphoma. Blood 129(4), 473-483 (2017).

5. Casulo C, Burack WR, Friedberg JW. Transformed follicular non-Hodgkin lymphoma. Am. Soc. Hematol. (2015). https://ashpublications.org/blood/article-pdf/125/1/40/1382839/40.pdf

6. Luminari S, Bellei M, Biasoli I, Federico M. Follicular lymphoma - treatment and prognostic factors. Associação Brasileira de Hematologia e Hemoterapia doi:10.5581/1516-8484.20120015 (2012). 
7. Matasar MJ, Luminari S, Barr PM et al. Follicular lymphoma: recent and emerging therapies, treatment strategies and remaining unmet needs. Oncologist 24(11), (e1236 -e1250 2019). https://pubmed.ncbi.nlm.nih.gov/31346132/

- Reports current landscape for treatment of follicular lymphoma and identifies the unmet need to understand first line treatments in practice.

8. Smith A, Crouch S, Lax S et al. Lymphoma incidence, survival and prevalence 2004-2014: sub-type analyses from the UK's Haematological Malignancy Research Network. Br. J. Cancer 112(9), 1575-1584 (2015).

-• Reports overall survival of various sub-types of hematological malignancy in UK.

9. Morrison VA, Shou Y, Bell JA et al. Treatment patterns and survival outcomes in patients with follicular lymphoma: a 2007 to 2015 humedica database study. Clin. Lymphoma, Myeloma Leuk. 19(4), e172-e183 (2019).

-• Reports data for follicular lymphoma treatment patterns and survival outcomes in the USA.

10. Ren J, Asche CV, Shou Y, Galaznik A. Economic burden and treatment patterns for patients with diffuse large B-cell lymphoma and follicular lymphoma in the USA. J. Comp. Eff. Res. 8(6), 393-402 (2019).

11. Albarmawi $\mathrm{H}$, Nagarajan $\mathrm{M}$, Sun $\mathrm{K}$ et al. Costs associated with follicular lymphoma among individuals diagnosed with non-Hodgkin lymphoma: a longitudinal analysis using SEER-Medicare data. Leuk. Lymphoma 61(1), 75-83 (2020).

- Reports a longitudinal analysis of follicular lymphoma patients using SEER-Medicare data in USA.

12. Rosenbaum PR, Rubin DB. The central role of the propensity score in observational studies for causal effects. Biometrika 70(1), 41-55 (1983).

13. International ethical guidelines for biomedical research involving human subjects. Bull. Med. Ethics (182), 17-23 (2002).

14. 'Consumer Price Index 2015-base consumer price index annual report yearly 2019 | file | browse statistics | portal site of official statistics of Japan'. https://www.e-stat.go.jp/en/stat-search/files?page=1 \&layout=datalist\&toukei=00200573\&tstat=000001084976\&cycle=7\&y ear $=20190 \&$ month $=0 \&$ tclass $1=000001085975$

15. Statistics Bureau of Japan. 'Statistics Bureau home page/consumer price index'. https://www.stat.go.jp/english/data/cpi/index.html

16. Zhang Z. Survival analysis in the presence of competing risks. Ann. Transl. Med. (2017). ttp://circ.ahajournals.org/lookup/doi/10.1161/CIRCULATIONAHA.115.017719

17. Austin PC, Lee DS, Fine JP. Introduction to the analysis of survival data in the presence of competing risks. Circulation 133(6), 601-609 (2016).

18. Terret C, Albrand G, Rainfray M, Soubeyran P. Impact of comorbidities on the treatment of non-Hodgkin's lymphoma: a systematic review. Expert Reviews Ltd (2015). https://pubmed.ncbi.nlm.nih.gov/25771832/

19. Nagai H. [Revision of JSH guideline for tumors of hematopoietic and lymphoid tissues 2018: lymphoma]. Rinsho Ketsueki 59(10), 2146-2152 (2018).

20. Gleeson M, Carolina R, Danese M, Knopf KB. Cost of follicular lymphoma (FL) in Medicare patients. J. Clin. Oncol. 26(Suppl. 15), 6634-6634 (2008)

21. Ministry of Health, Labor and Welfare of Japan. Various information of medical fee. Pharmaceutical Master Search 2020'. https://www.pmda.go.jp/files/000235661.pdf

22. Wang HI, Roman E, Crouch S et al. A generic model for follicular lymphoma: predicting cost, life expectancy and quality-adjusted-life-year using UK population-based observational data. Value Heal. 21(10), 1176-1185 (2018).

23. Soini EJO, Martikainen JA, Nousiainen T. Treatment of follicular non-Hodgkin's lymphoma with or without rituximab: cost-effectiveness and value of information based on a 5-year follow-up. Ann. Oncol. 22(5), 1189-1197 (2011).

24. Ray JA, Carr E, Lewis G, Marcus R. An evaluation of the cost-effectiveness of rituximab in combination with chemotherapy for the first-line treatment of follicular non-hodgkin's lymphoma in the UK. Value Heal. 13(4), 346-357 (2010).

25. Auweiler PWP, Mller D, Stock S, Gerber A. Cost effectiveness of rituximab for non-hodgkins lymphoma: a systematic review. Pharmacoeconomics (2012). https://pubmed.ncbi.nlm.nih.gov/22612993/

26. Hirayama $\mathrm{Y}$, Ishitani $\mathrm{K}$, Ota $\mathrm{S}$ et al. Long-term survey of survival time, histological transformation and secondary malignancies in Japanese patients with advanced-stage follicular lymphoma in the rituximab era: Hokkaido Hematology Study Group. Int. J. Hematol. 100(3), 281-289 (2014).

27. Monga N, Nastoupil L, Garside J et al. Burden of illness of follicular lymphoma and marginal zone lymphoma. Ann. Hematol. 98(1), 175-183 (2019).

28. Morrison VA, Bell JA, Hamilton L et al. Economic burden of patients with diffuse large B-cell and follicular lymphoma treated in the USA. Futur. Oncol. 14(25), 2627-2642 (2018).

-• Reports economic burden of Follicular lymphoma treatments in USA.

29. Beveridge R, Satram-Hoang S, Sail K et al. Economic impact of disease progression in follicular non-Hodgkin lymphoma. Leuk. Lymphoma 52(11), 2117-2123 (2011).

- $\quad$ Reports the burden of disease progression of follicular lymphoma patients. 
